\section{Symptomatic Hypotonic Hyponatremia Presenting at High Altitude}

\section{To the Editor:}

We appreciate that Spano et $\mathrm{al}^{1}$ have brought to your readers' attention an interesting case of symptomatic hyponatremia that was mistaken for high altitude cerebral edema. The case highlights the importance of recognizing overlapping signs and symptoms of exercise-associated hyponatremia (EAH) and acute altitude illness, as has been previously discussed. ${ }^{2,3}$ The Wilderness Medical Society Practice Guideline for treatment of $\mathrm{EAH}^{3}$ goes a step farther to point out that EAH may also be mistaken for dehydration or heat illness as a result of a similar complex of signs and symptoms. In general, incorrect diagnoses delay the institution of proper treatment. In the case of EAH, a delay in proper treatment can result in disastrous neurological sequelae. Even more concerning, though, can be treatment that could make matters worse, as it was with the case described by Spano et al. ${ }^{1}$

The case is that of a woman who had been hiking in the Sierra Nevada Mountains. During her second day, she experienced headache, nausea, vomiting, and diarrhea that was later followed by a grand mal seizure. She was incorrectly presumed to be suffering from high altitude cerebral edema and received at least $1.5 \mathrm{~L}$ of normal saline solution before recognition that she was hyponatremic. Isotonic and hypotonic fluids were continued after available physical examination and laboratory studies suggested that that she was hyponatremic and volume replete. Fortunately, it appears that likely effects from arginine vasopressin causing fluid retention had resolved, and her blood sodium was able to correct despite this intervention.

The current treatment recommendation for confirmed or suspected EAH is hypertonic saline solution rather than isotonic or hypotonic fluids. ${ }^{3}$ The hypertonic saline solution may be given orally when the patient can take oral fluids, but should otherwise be given as a $100-\mathrm{mL}$ intravenous bolus of $3 \%$ hypertonic saline solution, and repeated 2 additional times if needed. ${ }^{3,4}$ The rationale for concern about treating EAH with isotonic and hypotonic fluids has been extensively detailed. ${ }^{5}$ Although Spano et $\mathrm{al}^{1}$ comment that hypertonic saline solution has been used in the treatment of EAH, they lead the reader to believe that appropriate management of EAH is with isotonic fluids by the statements "normal saline therapy of symptomatic hypotonic hyponatremia can be effective [in this setting]" and "an initial normal saline bolus would be effective therapeutically in both hyponatremia and hypernatremia cases." Furthermore, they cite one of our publications to support the use of normal saline solution in treating EAH. That paper referred to 2 ultramarathon runners with combined biochemical hyponatremia and rhabdomyolysis progressing to acute kidney injury who received intravenous normal saline solution. ${ }^{6}$ We have discussed the importance of correctly balancing the contradictory interventions appropriate for EAH and acute kidney injury, and have recommended extreme caution in the use of intravenous normal saline solution as initial treatment when EAH may be a diagnosis. ${ }^{3,5-8}$ Acute kidney injury, as might occur in a volume-depleted state or as a consequence of rhabdomyolysis, was not a concern in the case described by Spano et al, ${ }^{1}$ so it cannot be used to rationalize use of isotonic and hypotonic fluids once the EAH diagnosis had been made.

The information provided in this case report suggests that the patient had overhydrated ("consumed liberal amounts of filtered water") and was not dehydrated given her admission blood pressure (although orthostatic blood pressures, which would have been most useful in assessing volume status, are not reported) in the face of normal blood urea nitrogen and creatinine concentrations. Her symptom of nausea, a potent nonosmotic stimulus for secretion of arginine vasopressin, was also likely involved in the initial pathogenesis of her severe hyponatremia. ${ }^{3,4}$ Interestingly, it is conceivable that the underlying stimulus for her nausea was acute mountain sickness. We do not believe there is support for a depletional mechanism to have played a role, as was suggested by the authors. Although a depletional mechanism may be involved in the development of EAH under some circumstances, ${ }^{7}$ as far as we are aware the severe symptomatic cases of EAH reported thus far in the literature have been associated with fluid overload without evidence for a depletional mechanism. ${ }^{4,7}$ The treatment for these cases of EAH should focus on elevating the blood sodium concentration rapidly to resolve cerebral edema, which can be achieved with a bolus of hypertonic saline solution without risk of osmotic demyelination or central pontine myelinolysis. ${ }^{3,4}$

Martin D. Hoffman, MD Department of Physical Medicine \& Rehabilitation Department of Veterans Affairs Northern California Health Care System and University of California Davis Medical Center Sacramento, $C A$

Robert H. Weiss, MD Department of Medicine Department of Veterans Affairs Northern California Health Care System Division of Nephrology 
Department of Internal Medicine University of California Davis Medical Center Sacramento, $C A$

\section{References}

1. Spano SJ, Reagle Z, Evans T. Symptomatic hypotonic hyponatremia presenting at high altitude. Wilderness Environ Med. 2014;25:69-74.

2. Ayus JC, Moritz ML. Exercise-associated hyponatremia masquerading as acute mountain sickness: are we missing the diagnosis? Clin J Sport Med. 2008;18:383-386.

3. Bennett BL, Hew-Butler T, Hoffman MD, Rogers IR, Rosner MH. Wilderness Medical Society practice guidelines for treatment of exercise-associated hyponatremia. Wilderness Environ Med. 2013;24:228-240.

4. Hew-Butler T, Ayus JC, Kipps C, et al. Statement of the Second International Exercise-Associated Hyponatremia Consensus Development Conference, New Zealand, 2007. Clin J Sport Med. 2008;18:111-121.

5. Bennett BL, Hew-Butler T, Hoffman MD, Rogers IR, Rosner MH. In reply to Clinical practice guidelines for treatment of exercise-associated hyponatremia. Wilderness Environ Med. 2013;24:468-471.

6. Bruso JR, Hoffman MD, Rogers IR, Lee L, Towle G, HewButler T. Rhabdomyolysis and hyponatremia: a cluster of five cases at the 161-km 2009 Western States Endurance Run. Wilderness Environ Med. 2010;21:303-308.

7. Hoffman MD, Hew-Butler T, Stuempfle KJ. Exerciseassociated hyponatremia and hydration status in 161-km ultramarathoners. Med Sci Sports Exerc. 2013;45:784-791.

8. Hoffman MD, Stuempfle KJ, Fogard K, Hew-Butler T, Winger J, Weiss RH. Urine dipstick analysis for identification of runners susceptible to acute kidney injury following an ultramarathon. J Sports Sci. 2013;31:20-31.

\section{In Reply to Symptomatic Hypotonic Hyponatremia Presenting at High Altitude}

\section{To the Editor:}

We would like to thank Doctors Hoffman and Weiss for their critique of our case report. Their letter references 2 valuable publications not cited in our work: the Wilderness Medical Society (WMS) Practice Guidelines for Treatment of Exercise-Associated Hyponatremia (EAH) ${ }^{1}$ and an excellent manuscript describing the overlap in clinical manifestations of EAH and acute mountain sickness (AMS). ${ }^{2}$ Neither of these resources articulates an actual case of hyponatremia mistaken for high altitude illness in the clinical setting, and this was the motivation for putting forth our case report. Furthermore, their letter takes to issue our treatment of hypoosmolar hyponatremia with isotonic fluids, which we will address below.
Treatment of hyponatremia can be challenging in a hospitalized patient and is even more challenging in the prehospital setting where diagnostic testing is limited. Although practice guidelines may offer explicit recommendations, they are based largely on expert opinion. Treatment decisions in individual cases often must be based on incomplete information and observed initial responses to therapy. When tailoring the appropriate response to individual patients, clinical uncertainty regarding the diagnosis being treated and the potential influence of anecdotal bias in the guideline development group need to be considered. The critique of our paper makes the assumption that we are reporting a case of EAH. We would like to challenge that assumption.

Our initial concern for altitude-related illness based on the clinical history was soon challenged by the finding of hyponatremia and, subsequently, by the magnetic resonance imaging (MRI), which failed to demonstrate cerebral edema. One could argue that had the low serum sodium been known earlier, an appropriate initial response by Parkmedics would have been hypertonic saline (HTS). However, the Parkmedics have neither the protocol nor the equipment to check prehospital sodium levels in Sequoia and Kings Canyon National Park. In this setting, the authors believe that isotonic saline was appropriate given the subacute onset ( $>48$ hours) of symptoms and the concern, based on the available history, for dehydration. Once in the hospital, the early observation of relatively highvolume, low-osmolality urine cautioned against a more aggressive approach to correction of her hyponatremia at that time.

If EAH has a similar pathophysiology as syndrome of inappropriate antidiuretic hormone (SIADH), as proposed by Bennett et al in the WMS Practice Guidelines, ${ }^{1}$ then the very low urine sodium is the most convincing objective evidence to support our position that this case was not one of simple EAH. In SIADH, body water is retained by the kidney at the expense of sodium because of the renal effects of antidiuretic hormone (ADH). One of the hallmarks of the diagnosis of SIADH should be urine sodium that is greater than $40 \mathrm{mEq} / \mathrm{L} .^{3-5}$ Our patient's urine sodium was nearly undetectable, indicating her kidneys were holding onto sodium, strongly arguing against an SIADH picture. The recent practice guidelines for the management of EAH do not address urine sodium as part of the initial evaluation, even though urine sodium less than $10 \mathrm{mEq} / \mathrm{L}$ suggests a good response to isotonic saline. ${ }^{5}$

Although we did not use invasive monitoring to confirm initial volume status, clinical indicators did not suggest our patient was in a fluid overloaded state. 